NECROLÒGICA 


\title{
Georges Duby: rigor y renovación de los estudios sobre edad media europea
}

\author{
Juan José Cortés García (coord.) \\ Universitat Autònoma de Barcelona \\ Institut d'Fstudis Medievals \\ 08193 Bellaterra (Barcelona). Spain
}

El 3 de diciembre de 1996, moría en su casa de Aix-en-Provence Georges Duby. La redacción de MEDIEVALIA se dispuso de inmediato, y con admiración, a reunir todos los testimonios que sobre su óbito se fueran produciendo a lo largo $\mathrm{y}$ ancho del mundo. Mirando las hemerotecas, pudimos comprobar la dimensión mundial que adquirió la resonancia de su muerte: una galería inmensa de retratos, opiniones, testimonios o confidencias se fueron publicando en los periodos y revistas especializadas. En esos días, decidimos reunir el material con vistas a una ulterior valoración. Eran los textos que mostraban el significado que Duby había tenido en la historia medieval de los últimos cincuenta años.

A continuación recogemos algunas de las opiniones vertidas en los períodicos al conocerse la noticia de su muerte. Jacques Le Goff: «Un nouveau Michelen (Liberation, 4/XII/96). Antoine Casanova: «Une parole toujours vivante» (L'Humanite, 4/XII/96). Michel Vovelle: «Explorateur de l'horizon humain" (L'Humanite, 4/XII/96). Michel Grodent "Un historien tout terrain» (Le Soir, 4/XII/96). Philippe-Jean Catinchi: «L'histoire comme une ouvre d'art" (Le Monde, 4/XII/96). Alain Peyrefite: "Le chevalier du Moyen Age" (Le Figaro, 4/XII/96). Emmanuel Le Roy Ladurie: "Un esprit généreux et désintéressés (Le Figaro, 4/XII/96); Chiara Frugoni "Signore dell'anno Mille» (La Repubblica, 4/XII/1996). Jean Daniel: «Con lui i secoli oscuri brillarono di luce» (La Repubblica, 4/XII/1996). J.E. Ruiz-Doménec: «El gran historiador del siglo XX1" (La Vanguardia, 5 de diciembre de 1996). José Antonio Millán: "El crucero de la historian (El Pais, 4/XII/1996); Uderico Munzi: "Il cavaliere del Medioevo". Douglas Jonhson: "The French historical revolution» (The Guardian, 4/XII/96). Octávio Gameiro: "Un dos mais importantes medievalistas do século XX” (Publico, 4/XII/96).

De este breve muestreo de opiniones expresadas en los periódicos como respuesta a la muerte, se puede entrever un retrato muy positivo, no sólo de Duby sino de toda la escuela francesa de historia en el proceso de renovación del estudio de la edad media, aunque a veces se lleve a cabo con un aire melancólico, como si la desaparición del "príncipen pudiera al terar el rumbo de la historio- 
grafia. Aprender a vivir sin él, profundizando en su legado, es una idea cambien generalizada, es probablemente el mensaje que expresa Le Goff, el más cercano y también el mejor situado para fijar la naturaleza de su trabajo.

La redacción de MEDIEVALIA, no satisfecha con estas primeras impresiones, quiso indagar el significado de la figura y la obra de Duby en tres importantes medievalistas actuales que mantuvieron con él relaciones de privilegio: Jacques Le Goff, sin duda el colega más cercano; Franco Cardini, el único no francés, que estuvo siempre cerca sin dejarse influir por sus métodos; Martin Aurell, el alumno y discípulo, primero en Aix-en-Provence y luego en París. A los tres les preguntamos lo mismo. Éstas fueron las preguntas de la redacción de Medievalia, y ésas fueron también sus respuestas:

Mèdievalıa: ¿Qué significado ha tenido la obra de Georges Duby para la historiografia en general? ¿Cudl ha sido su influencia en usted?

Jacques Le Goff: Georges Duby a renouvelé les études sur la féodalité et la Moyen Age dans le sens d'une approche interdiscipliaire que l'on peut placer sous la bannière de l'anthropologie historique. 11 a notamment élargi aux mentalités et aux représentations les conceptions héritées de Marc Bloch et des Annales. Ses recherches, ses articles et ses ouvrages m'ont conforté dans l'étude des mentalités et de l'imaginaire et le Dimanche de Bouvines, que je considere comme son chef d'oeuvre, m'a appris comment on pouvait unir l'étude de l'événement et celle des structures et de la longue durée.

Franco Cardini: Georges Duby appare come un maestro non legato in particolare ad al cuna scuola: e questo, sia chi aro, vuol essere un apprezzamento positivo. Dopo la sua fondamentale "tesi sul Maconnais", che resta un modello di ricerca «seriales, la sua personalitá di studioso del mondo medievale - - in particolar modo dei secoli XII-XIII- si è andata arricchendo e articolando fino a giungere a sintesi di grande rilievo e di grande profonditá, come i lavori sull'immaginario dell'etá feudale, sui rapporti tra il pensiero di Bernardo di Clairvaux e l'arte cirstercense, e a quel grande affresco di un'epoca che è Guglielmo Il maresciallo, accanto al quale porrei la monografia sulla battaglia di Bouvines. Due libri che hanno il rigore della ricerca storica e il fascino del romanzo: era dai tempi di Huizinga e di Bloch che non si scriveva nulla di simile. Per me, I'Antunno del medioevo di Huizinga, I re taumaturgi di Bloch e il Guglfelmo il Maresciallo di Duby sono stati tre libri fondamentali, senza i quali il nostro modo d'intendere il medioevo sarebbe diverso da quello che é; e senza i quali probabilmente io non avrei mai fatto neppure il "mestiere" di medievista. Il che, intendiamoci, può darsi sarebbe stato un bene per la stroai medievale.

Martin Aurell: La historiografía acaba de perder al mejor especialista actual del medioevo. Los casi 350 títulos de la obra de Georges Duby, iniciada en 1946, han marcado profundamente la investigación y las concepciones de numerosos historiadores. Empezó con la tesis sobre el Mâconnais, monografía regio- 
nal donde se utilizaban, casi por vez primera, sistemáticamente cartas monásticas para penetrar en una sociedad y una economía; la originalidad de la problemática, heredada sobre todo de Marc Bloch, y la claridad de expresión explican las reediciones sucesivas de un libro que no envejece. Después de haber publicado un corpus documental cisterciense (las pancartas de la abadía de la Ferté-sur-Grosne), G. Duby empieza lógicamente a hacer obras de síntesis, que satisfacen tanto al profesional de la historia como al hombre culto de la calle. Sobre todo, con etnólogos e historiadores de la escuela de los Annales, explora campos insospechados que le llevan de lo material (economía rural y vida campesina), a lo social (estructuras de parentesco o condición femenina), sin olvidar los valores y las «mentalidades” (le gustaba escribir esta palabra entre comillas). Fue un verdadero pionero.

Tuve la suerte de realizar mis estudios universitarios en Aix-en-Provence, donde Georges Duby ejerció la mayor parte de su docencia antes de ser elegido miembro del Collège de France. En Aix, había formado verdadera escuela, y sus discípulos - es decir mis profesores de primeros ańos de carreraelaboraban su tesis d'Etat o sus investigaciones sobre temas que él mismo les había señalado, a menudo, roturado; era como un maestro "mítico" del que me hablaban con veneración. A partir de cuarto de carrera pude seguir sus clases. En sus seminarios de doctorado en Aix y en París, aprendí que toda construcción histórica se fundamenta sobre una lectura atenta y minuciosa de los documentos, que él nos analizaba, desmontaba y comentaba con rigor. La lectura de sus obras, casi siempre de un tirón, me mostraba que estas mismas fuentes medievales son suficientemente ricas y explicitas: al buen historiador le basta resucitarlas, traducirlas en nuestra lengua, transferirlas a nuestra mentalidad y cuestionarlas con las problemáticas que preocupan a nuestros contemporáneos. Además, la calidad de escucha de G. Duby era proverbial; tenía confianza en los jóvenes investigadores, a los que nos cedía siempre la palabra en su seminario del Collège, delante de un areópago reunía a los mejores medievalistas franceses, europeos o americanos. Nos hacía creer en nuestras posibilidades, respetaba nuestro método y opciones historiográficas, nos animaba en los momentos dificiles que se pueden conocer a principios de una carrera de investigador, sin olvidar de corregir los errores provocados por nuestra inexperiencia. Fue un gran sabio de una erudición comunicativa y un verdadero maestro, apasionado por la enseñanza y por la transmision de conocimientos.

Medievalia: En la concepción de su discurso histórico destaca la conexión entre su forma de escribir y su interés por la divulgación de la bistoria a través de los medios de comunicación y de obras convertidas en éxitos editoriales. ¿Como valora las capacidades explicativas y/o descriptivas de su discurso en su tarea de acercar el conocimiento histórico al público?

J. Le Goff: Georges Duby nous a aussi montré qu'un grand historien pouvait et méme devait diffuser le résultats de ses recherches, et trouver une écriture qui leur assure un large public. Il nous a encouragés á sortir de la tour d'ivoi- 
re de l'érudition. Il a été á Aix et au Collège de France, un enseignant prestigieux qui a fait de nombreux disciples, par l'écriture accessible de ses ouvrages et par ses grandes réalisations télévisuelles ainsi que par son action á la présidence de la chaine ARTE, il a montré la voie de la vulgarisation de haut niveau aux érudits et aux universitaires.

F. Cardini: Sul rapporto tra ricerca scientifica e divulgazione i pareri degli studiosi sono controversi. Alcuni ritengono che la letteratura divulgativa sia sempre condannabile,e gli studiosi seri che vi si dedicano siano da giudicare con severicá: perché sprecano tempo che andrebbe dedicato alla ricerca, perché agiscono per leggerezza, per desiderio di una facile fama massmediale o per desiderio di guadagnar danaro. Altri al contrario praticano la divulgazione storica proprio per questi motivi. $\mathrm{Da}_{\text {a }}$ parte mia, ritengo la divulgazione storica nell'era dell' informatica, della telematica e della massmedializzazione un dovere civico da parte degli studiosi seri, La memoria storica é un bene troppo prezioso in un mondo minacciato dalla mondializzazione, dallí tribalizzazione e dalla polverizzazione individualistica: é necessario salvaguardara il nostro parimonio in termini d'identitá. D'altro canto, la divulgazione é una cosa tropp seria per lasciarla nelle mani dei divulgatori, che non hanno né la preparazione né l'onestá intellettuale di farla bene e che in generale si limitano a plagiare opere precedenti e a ridurre la storia a una galleria di curiositá aneddotiche. La divulgazione seria deve stabilire un filo diretto tra la ricerca scientifica seria e le capacitá intellettuali d'un pubblico il piú possibile vasto: il divulgatore serio dev'essere chiaro ma non banale, semplice ma non semplicista, aggiornato ma non pedante; deve possedere rigore scientifico e fascino di scrittura. Certo, tutto questo è difficile da trovarsi in una sola persona. Io oh scritto molte cose divulgative, ma mi ritengo un divulgatore mediocre. Georges Duby era un grande divulgatore: lo era perché era un grande storico e un grande scrittore

M. Aurell: Frase nominal, escansión breve, asíndesis, sinonimos en cascada, palabra preciosa, metáfora rebuscada, dramatización... el estilo de G. Duby es reconocible entre todos. Pero es mucho más que la simple forma que el medievalista intentaría dar a su discurso histórico. La escritura se transforma en historia misma. Como en poesía, la forma y el fondo, el significante y el significado, se mezclan en la obra del que pudo declarar: «Doy un precio enorme a la expresión, a la manera de escribir; ante todo, juzgo la historia esencialmente como un arte literarion. Fascinado por la pintura y la escultura contemporáneas, G. Duby encontró en el manejo de las palabras la misma sensación estética, la misma intuición poética que el artísta en las imágenes y en las formas creadas. Esta calidad de expresión se explica por el ambiente cultural en que se desarrolló su infancia, entre maestros de escuela de principios de siglo, para Jos que el cul ro de la claridad, de la lógica y de la razón aparecía como la forma suprema del genio francés («o que se concibe bien, se expresa claramenten, Ce qui se convoit bien s'exprime cluirement, dice el adagio galo) y en una familia de artesanos en la que la obra maestra, fruto de un 
trabajo perfeccionista, era el ideal y el orgullo de un gremio. Gracias a esta cuidada retórica, el discurso histórico de G. Duby es asequible a auditores y lectores de horizontes tan disparatados.

MEDievala: Una constante de su cjercicio como bistoriador ba sido la utilización de una gran diversidad de fuentes desde una perspectiva interdisciplinar. ¿Qué repercusión considera que ha tenido para la disciplina histórica?

J. Le Goff: Malgré l'importance du cartulaire de Cluny pour l'élaboration de sa thése, Georges Duby a voulu montrer qu'un probléme historique ne nous réduisait pas á l'exploitation d'un type de sources comme c'était trop souvent le cas dans l'histoire traditionnelle. Il a élargi le territoire et l'appétit de l'historien.

F Cardini: L'interdisciplinarietá é divenuta ormai necessaria. Ciò sottintende che le vecchie divisioni e le vecchie differenze tra gli studiosi non reggono più, sono crollate. Ma oggi viviamo nel mondo della storia (e forse in tutto il mondo della ricerca scientifica) una drammatica contraddizione, un vero paradosso. Da una parte diventa sempre piú importante specializzarsi ad alto livello, quindi in campi sempre piú limitati del sapere; ma ciò rischia di condurci verso forme di iperspecializzazione incapaci di comunicaré tra loro: é il rischio di una certa americanizzazione della ricerca scientifica. Dall'altra si ha crescente bisogno di collegare e di coordinare tra loro ámbiti e aspetti diversi della ricerca, con il pericolo di un neoenciclopedismo che potrebbe prédisporre alla superficialitá (ed é un pericolo che l'informatizzazione della ricerca rende piú grave). La via d'uscita, forse, sta nella ricerca di équipe, senza però farsi grandi illusioni. Quando si tratta di grandi intuizioni, lo studioso è sempre solo con se stesso. Georges Duby ha contribuito, con Fernand Braudel, con Jacques Le Goff e con molti rappresentanti della nouvelle histotre, ad aprire le porte allo stretto rapporto tra storia e scienze umane. Questo è un grande merito, che ha messo la storia in grado di compiere importanti progressi.

M. Aurell: Como Marc Bloch y Lucien Febvre le habían enseñado, todo puede ser objeto histórico. No existen fronteras ni muros entre las ciencias humanas que investigan sobre el hombre en el pasado. La interdisciplinariedad fue un credo para G. Duby: la ejercitó sobre todo con etnólogos; la comunidad científica se acuerda de la emoción con que recibió su espada de académico de manos de Claude Lévi-Strauss. Como consecuencia de esta ampliación del campo de la erudición histórica, utilizó profusamente las fuentes literarias o artísticas, que situó en su contexto social, político y mental. Se necesitaba valentía porque este "anexionismo", que todo historiador sólo puede aprobar, no fue siempre del gusto de otros investigadores: algunos literarios le criticaban de leer más los textos en su "contexto" que en su cintertextualidado; los historiadores del arte le echaban en cara de no dar a la obra su verdadera dimensión artística, puesto que no la consideraba como un todo autónomo, como 
una finalidad en sí misma. Olvidaban, de hecho, la gran sensibilidad de G. Duby por el arte - él mismo pintó con acierto - y su amistad con numerosos pintores o escultores actuales. Una sensibilidad idéntica se encuentra en su percepción del fenómeno religioso, espirirual, que nunca rechazó, aunque por supuesto lo sabía encarnado, condicionado por la cultura y la civilización en el que se manifiesta.

Medievalia: La reflexión histórica de Georges Duby sobre la sociedad feudal se ha centrado principalmente en tres grandes problemas: los sistemas de valores, el imaginario y las mujeres. Valore el alcance de sus aportaciones.

J. Le Goff: Georges Duby a profondément transformé et enrichi le regard porté sur la féodalité) en plagant les mentalités et les comportements au coeur du fonctionnement du systéme féodal comme le montrent ses études sur les jeunes et ses travaux sur la mentalité chevaleresque évoquée dans un Guillaume le Maréchal, 2) en mettant en valeur plus, me semble-t-il, l'idéologie que l'imaginaire car tel est bien l'objet du grand livre sur les Trois ordres malgré son soustitre oú idéologie conviendrait mieux qu'imaginaire.

Sur la place des femmes dans la société et le systéme féodal, et sur la dificulté que nous avons á les entendre parce que la parole ne leur a guére été donnée dans les sources, il a ouvert un vaste territoire dans l'étude de la société féodale, Georges Duby a créé une oeuvre qui illustre bien l'ambition rarement réalisée par d'autres d'une histoire totale dans la ligne du mouvement des Annales et son talent d'écriture a doublé les leçons d'un grand historien des prestiges d'un grand écrivain.

F. Cardini: Per quel che riguarda l'immaginario feudocavalleresco, credo che gli studi sulla cavalleria di Georges Duby -insieme con quelli di Erich Koelher, di Joël Grisward, di Jean Flori e di altri abbiano davvero rivoluzionato e rinnovato quello specifico campo di studi. Ma ritengo che all'origine di questo rinnovmento vi sia la forte influenza delle ricerche di Georges Duméxil, delle quali io stesso sono debitore. Studiando chierici e cavalieri, specie in quel xII secolo che, --come dice Denis de Rougemont- ha inventato llamore, Duby si é incontrato con il problema femminile in tutte le sue espressioni: dal culto della Vergine Maria al ruolo sociale e civile delle donne nel medioevo. In ciò il suo lavoro si è andato negli ultimi anni intrecciando al crescere quantitativo e spesso (non sempre) anche qualitativo delle ticerche dedicate alla estoria delle donnes. Qui mi domando tuttavia se, per ben valutare llapporto positivo del Duby, dovremo aspettare che passi una certa moda di studi connessi un po'troppo strettamente con ta uni aspetti del mondo e della mentalitá "femministis, nonché con le ambiguitá relative a quelli che gli statunitensi chiamano i gender studies.

M. Aurell: Duby vivió con su tiempo; su curiosidad hacia todas las ciencias humanas le hizo seguir caminos muy diversos, los derroteros seńalados por las 
diferentes "modas intelectuales parisinas" (sin intención peyorativa alguna, sino con orgullo) de los años 45-95. La evolución de sus centros de interés se explica por un contexto de inquietud de pensamiento y de de cambio continuo de sensibilidad que caracteriza nuestro siglo. Su primera fascinación por el marxismo, tan influyente en la escuela francesa de aprés-guerre, le llevó hacia el estudio de la civilización material, de las técnicas y de las relaciones entre señores y campesinos, aunque sin adoptar un análisis determinista de las relaciones entre infraestructuras y superestructuras. Luego, la antropologia cultural le inició en problemas de parentesco y de alianza. Se sitúan en el campo de estos trabajos sus investigaciones sobre la mujer, aunque potencializadas por la Gender History ultraatlántica de los años 70. Pero gracias al descubrimiento de la historia de las mentalidades, que él mismo animó con entusiasmo, analizó con su finura habitual los mecanismos de la ideología y del imaginario político medievales. Cómo no aludir a su Bouvines, obra maestra construida entorno a una batalla, un acontecimiento, algo tan criticado por los fundadores de los Annales ! Había que ser iconoclasta para empezar con énfasis este libro por un «relato", forma de discurso tan específicamente historiográfico. Su inventiva y su capacidad de adaptación fueron ejemplares. Como él, todo historiador debería saber cuestionarse, autocriticarse cada día, no conformarse nunca con lo que ha adquirido, sino lanzarse continuamente en investigaciones innovadoras. Sólo asi conservó $\mathrm{G}$. Duby su gusto por los archivos y por la historia en una juventud perenne. Éste parece ser su principal legado.

Para valorar en toda su dimensión estas opiniones, la redacción de MEDIEvALLA ofrece al lector español un currículo abreviado de su vida y obra:

París, Francia, 7 de octubre de 1919 - Aix-en-Provence, Francia, 3 de diciembre de 1996.

\section{Formación}

Lycée a Mâcon.

Geografia e Historia en la Facultad de Letras de la Univerdidad de Lyon.

Defensa de su tesis doctoral dirigida por Charles-Edmond Perrin en la Universidad de La Sorbona. París, 1953.

\section{Trayectoria}

Profesor agregado en la Univerdidad de Lyon, 1942.

Profesor asistente de Historia de la Edad Media en la Facultad de Letras de la Universidad de Lyon, 1945.

Profesor de la Facultad de Letras de la Universidad de Besançon, 1950-1951. Profesor de la Facultad de Letras de la Universidad de Aix-en-Provence, 1951. Cátedra de Historia de la Edad Media en la Facultad de Letras de la Universidad de Aix-enProvence 1953. 
Juan José Cortés Garcia (coord.)

Cátedra de Historia de las Sociedades Medievales del Collège de France, 1970.

Miembro del consejo editor de la cadena televisiva SEPT, 1986-1988.

Presidente de la cadena televisiva SEPT entre 1988 y 1993.

\section{Bibliografia:}

\section{Libros:}

La Société aux Xle et XII siècles dans la région màconnaise, SEVPEN, 1953.

Recueil des pancartes de l'abbaye de la Ferté-sur-Grosne, 11 13-1178. Aix-en-Provence, 1953.

Histoire de La civilisation française, vol 1. Colin, 1858.

L'Economic rurale, et la vie des campagnes dans l'Occident médiéval. Aubier, 1962, con R. Mandrou (Trad. castellana).

L'Europe des cathédrales, 1140-1280. Albert Skira, 1966.

Fondements d'un nouvel humanisme, 1280-1440. Albert Skira, 1966.

Adolescence de la chrétienté occidentale, 980-1140. Albert Skira, 1967.

L'An mil. Colección "Archives*. Julliard, 1967 (Trad. castellana).

Medieaeval Agriculture, 900-1500, vol. I de The Fontana Economic History of Europe. Collins, 1971.

"Les societés médiévales, une approche d'ensemble». Lección inaugural en el College de France pronunciada el 4 de diciembre de 1970. Gallimard, 1972.

Guerriers et paysans. Gallimard, 1973 (Trad. castellana).

Le Dimanche de Bowvines. Gallimard, 1973 (Trad. castellana).

Les Procés de Jeanne d'Arc. Colección "Archives" Julliard-Gallimard, 1973, con Andrée Duby.

Les Temps des cathédrales. L'art et la societe, 980-1420, Gallimard, 1976. (Trad.castellana).

Hommes et structures du Moyen Age. Mouton-EPHE, 1973 (Trad. castellana).

Saint Bernard. L'art cistercien. Arts el Métiers graphiques, 1976. (Trad.castellana).

Georges DUBY-Jacques LE GoFF: Famille et parenté dans l'Occident médiéval. Boccard, 1977.

Mediaeval Marriage. Two models from Twelfh-Century France. Baltimore-Londres, 1978.

Les Trois Ordres ou l'imaginaire du féodalisme. Gallimard, 1978 (Trad. castellana). L'Europe au Moyen Age. Art roman, art gothique. Arts et Métiers graphiques, 1979 (Trad. castellana).

Dialogues avec Guy Lardreau. Flammąion, 1980.

Le Chevalier, la femme et le prêtre: Le Mariage dans la France féodale. Hachette, 1981. (Trad. castellana).

L'Esurasie (11-13 siècles). PUF, 1982.

Guillaume le Maréchal ou le Meilleur Chevalier du monde. Fayard, 1984 (Trad. castellana).

Le Moyen Age. Albert Skira, 1984.

Fernand BRAUCIEL-Georges DUBY: La Mediterranée. Les hommes et l'héritage. Flammarion, 1986 (Trad, castellana).

Male Moyen Age. De l'amour et autres essais. Flammarion, 1988 (Trad. castellana). La Sculpture. Skira, 1990.

L'Histoire continue. Odile Jacob, 1991 (Trad. castellana). 
Georges Duby-Bronisiaw Geremek: Passions comnunes. Seuil, 1992.

Femmes et bistoire. Plon, 1993.

La Chevalerie. Perrin, 1993.

An 1000 An 2000. Sur les traces de nos peurs. Textuel, 1995 (Trad. castellana).

Dames du XIse siécle I. Héloise, Aliénor, Iseut et quelques autres. Gallimard, 1995. (Trad. castellana).

Dames du XIIe siècle II. Le souvenir des aïeules. Gallimard, 1995 (Trad.castellana).

Le Moyen Age. Seuil, 1995.

Féodalite. Collection "Quarton, Gallimard, 1996.

\section{Articulos}

Recherches sur l'evolution des institutions judiciaires pendant le Xe siècle dans le sud de la Bourgogne *. Le Moyen Age, 52. 1947.

"Economie domaniale et économie monétaire. Le budget de l'abbaye de Cluny entre 1080 et 1155 w. Annales ESC. abril-junio de 1952.

«Dangers d'une réussite» Saint Bernard homme d'Eglise. Cahiers de la Pierre-quivire. Paris, 1953.

"Un inventaire des profits de la seigneurie clunisienne à la mort de Pierre leVénérable». Studia Anselmiana (40), 1956.

"La structure d'une grande seigneurie flamande à la fin du XII siècle». Biblioteque de L'Ecole des Chartres, 1956.

"Techniques et rendements agricoles dans les Alpes du sud en 1338*. Annales du Midi, 1958.

*Métamorphose d'André Masson». L'Arc, 2, abril de 1958.

"La féodalité? Une mentalité médiévale, une enquête à poursuivre», Annales ESC. 13, octubre-diciembre de 1958.

«Note sur les corvées dans les Alpes du Sud en 1338”. Entudes d'histoire de droit privé offertes à Pierre Petot. París, 1959.

"Les villes du sud-est de la Gaule du vitle au Xıe siècle», La città nell' alto medioevo. Spoleto, 1959.

"La seigneurie et l'économie paysanne, Alpes du Sud 1338n. Érudes rurales, 2, julioseticmbre, 1961.

"Une enquête a poursuivre: la noblesse dans la France Medievale». Revue Historique, 226. 1961.

¿Les canoniques réguliers et la vie economique aux XIe et XIle siècles». La vita comune del clero nei secoli XIe XII. Milán. Societá Editrice Vita e Pensiero, 1962. p. 72. 81 .

"Dans la France du Nord-Ouest, au XIle siècle: Les "jeunes" dans la societé, aristocratique». Annales ESC, 19, setiembre-octubre de 1964.

"Les laücs et la paix de Dieu* I lä̈ci nella ssocietas christiana" dei secoli XI e XII. Milán, 1966.

"Le probléme des techniques agricoles". Agricoltura e mondo rurale in occidente nell" alto medioevo. Spoleto, 1966.

"Structures de parenté el noblesse. France du Nord, XIe-XIle siècles 2w. Miscellanea mediaevalia in memoriam Jan Frederik Niermeyer. Groningue, 1967.

«Remarques sur la littérature généalogique en France aux xle et Xne siècles». Academie des Inscriptions et Belles Lettres Comptes Rendus des séances. París, 1967. 
"La vulgarisation des modèles culturels dans la sociéte féodalen. Niveanx de culture el groupes sociaux: actes du colloque réuni du 7 au 9 mai 1966 à l"Ecole normale supérieure. Mouton, 1967.

"L'Histoire des mentalités". L'Histoire et ses méthodes. Encyclopédie de la Plétade, 1967. "Structures familiares aristocratiques a la France au XI siècle en relation avec les structures de l'Étato. Actas del coloquio Europa durnte los siglos DX-XZ. Varsovia-Poznan, 1967.

"Les origines de la chevalerien. Ordinamenti militari in Occidente nell'alto medioevo. Spoleto, 1968.

*Hérésies et sociétés dans l'Europe pré-industrielle. $11 \mathrm{e}-18 \mathrm{e}$ siècles. Conclusion». Collogue de Royaumont. Mouton-Ecole Pratique des Hautes Études. 1968.

"Situation de la noblesse dans la France à le commencement du XIII sic̀clen. Tijdschritt voor Geschiedenis. 1969, p. 309-315.

«Structure familiale dans le Moyen Age occidentals, XII Congreso internacional de ciencias históricas. 16-23 de agosto de 1970. Académie des Sciences d'URSS. Moscú, 1970.

"Histoire et sociologie de l'occident médiévale. Conclusions et investigations».Revue roumaine d'Histoire 9(3), 1970, p. 451-458.

"Le monaquisme et l'économie rurale». Il monachesimo e la riforma ecclestastica, 1049 1122. Atri della quarta Setrimana internazionale di Studio. Mendola, 23-29 de agosto de 1968. Milán. Editrice vita e Pensiera, 1971, p. 336-349.

«Lignage, noblesse et chevalerie au XIle siècle dans la région maconnaise, une révisions. Annales ESC, julio-octubre 1972.

«L'image du prince en France au XIe siècle». Cabiers d Histoire, 1972.

¿L'Histoire des systemes de valeurs». Studies in the Philosophy of History, vol. XI, n. I. 1972. Wesleyan University Press.

"Entretien avec A. Casanovav. Aujourd'bui l'Histoire. Editions sociales, 1974.

-Histoire sociale ex idéoiogies des sociétés". Faire de l'Histoire, vol. 1. Gallimard, 1974. Prólogo a Eaux Forres de Soulages. Arts el Métiers graphiques, 1974.

-La diffusion du titre chevaleresque sur le versant méditerranéen de la chrétienté latinev. La noblesse au Moyen Age. Paris, 1976.

"Gérard de Cambrai, la paix et les trois fonctions sociales, 1024" Academie des Inscriptions et Belles Lettres Comptes Rendus, 1976.

Prólogo a la edición del Roman de la rose. Club du livre, 1976.

"Le mariage dans la societé du Haut Moyen Agex. Il matrimonio nella societá altomedievale. Spolero, 1976.

„Mémoire sans historien". Nouvelle Revue de psychanalyse. XV, primavera de 1977. Prólogo a Fêtes en France. Ed. du Chène, 1977.

"Problèmes et Mćthodes de l'histoire Culturel». Actas del coloquio Objeto y métodos de la historia de la cultura. Tihany, 10-14 de octubre de 1977.

"Le mental et le fonctionnement des sociétés bumaines», Georges Duby. L'Arc, 72, 1978.

"Les transformations sociales dans le milieu aristocratiquen. La france de Philippe Auguste, Le temps des mutations. Coloques Internacionales CNRS, 1982.

"Audience and Patronagen. Renasissance and Renewal in twelfth century. Harvard Cambridge, 1982.

«The Matron and the Mis-Married Woman: Percepcions of Marriage in Nothern France "circa" 1100 ». Social and Ideas: Essays in Honour of R.H. Hilton. Aston, Coss, Dyer and Thirsk. Past and Present Society, 1983. 
"Un entretien avec Georges Duby sur la littérature courtoise», Ornicar, 26-27. 1983. "Que sait-on de l'amour en France au Xlle siècle?". The Zaharoff Lecture for 19821983. Nueva York-Oxford, 1983.

Prólogo a La memoria de los feudales de J. E. Ruiz Doménec. Argot, 1984.

"Por una historia de las mujeres en Francia y en Espańa. Conclusiones de un coloquio.» Coloquio La Condición de la mujer en la Edad Media. Madrid, 1986. Ed. Universidad Complutense, Madrid, 1986.

"Réflexions sur le douleur physique dans le Moyen Ages. Coloquio organizado por la Universidad de Varsovia. 1986. Revue des sciences médicales $n^{\circ} 345,1986$.

«A propos de l'amour que l'on dit courtoiss. Académie royale de langue et de littérature française. Bruselas, 13 de diciembre de 1986.

"Le plaisir de l'historien". Essais d'ego-histoire. Gallimard, 1987.

Prólogo a la edición de La Chanson de la Croisade albigeoise. Colección "Lettres Gothiques». LGF, 1989.

\section{Dirección de obras colectivas}

Histoire de France. Larousse. 1982.

Histoire de la France rurale. Seuil, 1975-1978.

Atlas historique. Larousse, 1978.

Histoire de la france urbaine. Seuil, 1980-1983

Histoire de la vie privée. Plon, 1985.

Civilisation latine. Orban O, 1986.

Histoire des femmes. Plon, 1991.

Histoire artistique de l'Europe. T. 1 Le Moyen Age, Seuil, 1995.

\section{Miembro de instituciones}

British Academy.

Académie Royale de Belgique.

Royal Historical Society.

Medieval Academy of America.

Accademia Nazionale dei Lincei (Roma).

Real Academia de Buenas Letras de Bareclona.

Academia de Lengua y Literatura Francesas de Bélgica.

Academia de Ciencias de Hungría.

American Philosophical Society (Filadelfia).

Académie des inscriptions et Belles-Lettres, 1974.

Académie Française, 1987.

Miembro del Consejo Superior de la Lengua Francesa, 1989.

Consejo Científico de la Fundación Francesa.

\section{Distinciones}

Comendador de la Legion d'Honneur de las Artes y las Letras.

Honoris causa por Oxford.

Honoris causa por Santiago de Compostela, 1992. 
Premio Gobert en 1962 por su obra LEconomie rurale et la vie des campagnes dans l'Occident médieual.

Premio de la Fondation de France en 1973 por su obra Guerriers et paysans.

Premio Ambassadeurs en 1973 por su obra Le Dimange de Bouvines,

Premio Gutemberg en 1985 por su obra Historia de la vida privada.

Escribir de otra manera la historia, en ello estribó la grandeza de Duby. Su legado es una utopía de renovación; en la que el rigor no está reñido con la amenidad. La grandeza de su persona es que, al fin y al cabo, hizo historia escribiendo sobre ella; sólo así, protagonista y escritor, pudieron fundirse en un mismo individuo que miraba el mundo de forma aguda. Eso se observa en sus últimos trabajos, cuando habla del futuro, y no del pasado, donde una conciencia trágica emerge de la sensibilidad por el estudio de un período lejano y dificil. Desde esta redacción pedimos por él: descanse en paz ${ }^{1}$.

1. En el momento de esta redacción han aparecido dos importantes necrológicas. Son las siguientes:

Jacques LE GoFF, "Nécrologie: Georges Duby (1919-1996)", en Cabiers de Civilisation Medievale, XI e et xile siècles, 40e Année-Avril-Juin, Poitiers, 1996-1997, p. 199-209.

José Enrique RUIZ DOMENEC, * Una mirada sobre Dubys, en BRABLB 1996, p. 493-505. 